\title{
Erratum to: Sustainable Energy Technology and Policies
}

Sudipta De, Santanu Bandyopadhyay, Mohsen Assadi and Deb A Mukherjee

\section{Erratum to:}

\section{S. De et al. (eds.), Sustainable Energy Technology and Policies, Green Energy and Technology, https://doi.org/10.1007/978-981-10-7188-1}

The original version of the book was inadvertently published with incorrect Foreword version which has been now updated with correct version in Frontmatter. The erratum book has been updated with the change. 